\title{
Influence of time under mechanical ventilation on bronchopulmonary dysplasia severity in extremely preterm infants: a pilot study
}

Victoria Escobar ${ }^{1,2}$, Darllyana S. Soares ${ }^{1,2}$, Jane Kreling ${ }^{1}$, Ligia S. L. Ferrari, ${ }^{1,3}$, Josiane M. Felcar ${ }^{4,5}$, Carlos A. M. Camillo $0^{2,4,5}$ and Vanessa S. Probst ${ }^{2,4^{*}}$ (D)

\begin{abstract}
Background: The relation between mechanical ventilation (MV) and bronchopulmonary dysplasia (BPD) - a common disease in extremely premature newborn (PTNB) - is well stabilished, but is unknown, however, how much time under MV influences the severity of the disease.

Aim: To define the duration under MV with greater chance to develop moderate to severe BPD in extremely PTNB and to compare clinical outcomes before and during hospitalization among patients with mild and moderate to severe BPD.

Methods: Fifty-three PTNB were separated into mild and moderate to severe BPD groups and their data were analyzed. Time under MV with a greater chance of developing moderate to severe BPD was estimated by the ROC curve. Perinatal and hospitalization outcomes were compared between groups. A logistic regression was performed to verify the influence of variables associated to moderate to severe BPD development, such as pulmonary hypertension (PH), gender, gestational age (GA) and weight at birth, as well the time under MV found with ROC curve. The result of ROC curve was validated using an independent sample $(n=16)$ by Chi-square test.
\end{abstract}

Results: Time under MV related to a greater chance of developing moderate to severe BPD was 36 days. Moderate to severe BPD group had more males ( 14 vs $5, p=0,047$ ), longer time under MV (43 vs 19 days, $p<0,001$ ), more individuals with PH (12 vs 3, $p=0,016$ ), worse retinopathy of prematurity (grade 3, 2 vs $11, p=0,003$ ), longer hospital length of stay (109 vs 81,5 days, $p<0,001$ ), greater PMA (41 vs 38 weeks, $p<0,001$ ) and weight (2620 vs $2031 \mathrm{~g}, p<0,001$ ) at discharge and the mild BPD group had more CPAP use prior to MV (12 vs 7, $p=0,043)$. Among all variables included in logistic regression, only $\mathrm{PH}$ and $\mathrm{MV}<36$ days were significant in the model, explaining $72 \%$ of variation in moderate to severe BPD development. In the validation sample, prevalence of preterm infants who needed MV for more than 36 days in the moderate to severe BPD group was 100\% $(n=6)$ and $0 \%$ in mild BPD group $(p=0,0001)$.

\footnotetext{
* Correspondence: vanessaprobst@gmail.com

${ }^{2}$ Postgraduation Program in Rehabilitation Sciences UEL-UNOPAR, Londrina State University (UEL), Londrina, PR, Brazil

${ }^{4}$ Physiotherapy Department, Center of Health Sciences, Londrina State University, 60 Robert Koch Av, Londrina, Parana 86038-350, Brazil

Full list of author information is available at the end of the article
}

C C The Author(s). 2020 Open Access This article is licensed under a Creative Commons Attribution 4.0 International License, which permits use, sharing, adaptation, distribution and reproduction in any medium or format, as long as you give appropriate credit to the original author(s) and the source, provide a link to the Creative Commons licence, and indicate if changes were made. The images or other third party material in this article are included in the article's Creative Commons licence, unless indicated otherwise in a credit line to the material. If material is not included in the article's Creative Commons licence and your intended use is not permitted by statutory regulation or exceeds the permitted use, you will need to obtain permission directly from the copyright holder. To view a copy of this licence, visit http://creativecommons.org/licenses/by/4.0/. The Creative Commons Public Domain Dedication waiver (http://creativecommons.org/publicdomain/zero/1.0/) applies to the data made available in this article, unless otherwise stated in a credit line to the data. 
(Continued from previous page)

Conclusion: Time under MV related to moderate to severe BPD development is 36 days, and worst outcomes are related to disease severity. PH and time under MV for more than 36 days are related to development of moderate to severe BPD.

Keywords: Premature, Bronchopulmonary dysplasia, Mechanical ventilation

\section{Background}

Bronchopulmonary dysplasia (BPD) is a multifactorial disease that occurs due to interactions among exposures during pregnancy, injuries from oxygen use and postnatal invasive mechanical ventilation (MV), as well as other injuries such as infections and inadequate nutrition after birth [1]. Diagnosis and classification remain uncertain, as there is still no consensus on the ideal postmenstrual age (PMA) for assessment of oxygen-dependent premature newborns. The 2000 National Heart, Lung and Blood Institute workshop recommends that for preterm infants with gestational age less than 32 weeks, BPD is defined as oxygen exposure for $\geq 28$ days. It can also be categorized as mild (in room air at 36 weeks of PMA or at discharge whichever comes first), moderate (supplemental oxygen at 36 weeks of PMA or at discharge - whichever comes first), or severe (supplemental oxygen $\geq 30 \%$ and/or positive pressure at 36 weeks of PMA) [2]. BPD is a morbidity associated with extreme prematurity, and it has now been debated that milder forms of BPD do not seem to have severe consequences, such as serious respiratory morbidity and neurosensory impairment $[1,3]$.

Extremely preterm infants, those born at $<28$ weeks of PMA [4], are frequently submitted to MV [5], which despite being a necessary and widely used treatment, has well established adverse effects. The risk of death is 8 times higher in extremely low birth weight preterm infants undergoing MV for more than 6 weeks compared to those exposed only to 7 days or less of invasive ventilation. This risk increases to 13 times when isolated only to cardiorespiratory causes [6]. Morbidities such as pneumothorax, ventilator-associated pneumonia, retinopathy of prematurity (ROP) requiring surgical intervention and even neurodevelopmental impairments are also associated with MV in preterm infants [7-9]. In addition, duration of $\mathrm{MV}$ is a strong predictor for BPD development: each additional week increases 2.7 times the odds for BPD [10]. Although the relationship between invasive mechanical ventilation and the development of the disease is well established, the duration of MV that interferes with severity classification of BPD remains unknown.

Therefore, the aim of this study was to determine how many days of mechanical ventilation has a greater chance of developing moderate and severe bronchopulmonary dysplasia. Moreover, we aimed to compare clinical variables during hospitalization and prenatal data between patients with mild and moderate to severe dysplasia.

\section{Methods \\ Design and sample}

This was a retrospective cohort study (with clinical data prospectively entered in a national database) which was conducted at the University Hospital of the Londrina State University - Brazil. All preterm infants born at $\leq 28$ weeks PMA between January 2015 and December 2017, admitted to the neonatal unit who survived for more than 28 days and were diagnosed with BPD were eligible. Infants who did not use invasive mechanical ventilation during hospitalization and those who died before 36 weeks of PMA were excluded. This was considered as a pilot study since we assessed data from only one hospital which participates in the Brazilian Neonatal Research Network. The study was approved by the Research Ethics Commitee Involving Human Beings Londrina State University - Brazil (commitee's reference number: 3.362.155).

\section{Procedures}

Patients included in the study were allocated into two groups: (I) mild BPD, those with diagnosis of BPD breathing in room air at 36 weeks of PMA; and (II) moderate to severe BPD, preterm infants diagnosed with $\mathrm{BPD}$ who required oxygen or some type of ventilatory support at 36 weeks of PMA. The definition of BPD and its severity were described by Jobe and Bancalari [2].

\section{Perinatal variables were didactically divided into four groups}

a) Maternal and gestational characteristics: use of tobacco during pregnancy, use of antenatal corticosteroids and clinical diagnosis of chorioamnionitis;

b) Perinatal variables: postmenstrual age - weeks and days of gestation determined by ultrasound in the first trimester or date of last menstruation - birth weight, gender, Apgar score at 5th minute of life and type of stabilization required in the delivery room - intubation or noninvasive ventilation through continuous positive airway pressure CPAP;

c) Outcomes during hospitalization: time under invasive mechanical ventilation, CPAP use before intubation, surfactant admnistration, vasoactive drugs (VADs) until the third day of life, degree of 
retinopathy of prematurity [11] and need for surgical correction - diagnosed by an ophthalmologist by retinal examination, patent ductus arteriosus (PDA) - diagnosed by a pediatric cardiologist by echocardiography - and type of treatment - drug or surgical, also indicated by cardiologist, pulmonary hypertension (PH) - also diagnosed by a pediatric cardiologist on echocardiography - clinical diagnosis of necrotizing enterocolitis (NEC) [12], pneumonia, pneumothorax and sepsis [13];

d) Variables at discharge: length of stay, weight and PMA at discharge.

\section{Data on invasive mechanical ventilation}

The two main ventilation modes used in the study were time-cycled pressured-limited continuous flow ventilation and target volume ventilation. High-frequency oscillatory ventilation was rarely used. Extubation criteria included arterial blood gases within normal limits and ventilator parameters that were considered low, in addition to the stability of the newborn.

\section{Statistical analysis}

Statistical analysis was performed using SPSS Statistics 22 (IBM Corp, Armonk, NY) and Graph Pad Prism 6.0 software (GraphPad Software Inc; San Diego, California, USA). Data distribution analysis was performed by Shapiro-Wilk test and data were described as percentages and mean \pm standard deviation or median and interquartile range, according to the normality test. To estimate time under mechanical ventilation to predispose the highest chance of developing moderate to severe BPD, a ROC curve with time under mechanical ventilation was generated in days for both groups, and the best combination of sensitivity and specificity was used (index of Youden [14]). The area under the ROC curve (AUC) is a measure of cutoff point accuracy. AUC ROC values $\geq 0.90$ are considered excellent, $0.80-0.89$ good, $0.70-0.79$ reasonable and $<0.70$ are considered poor [15]. Comparison of variables between groups was performed by Student's t-test or MannWhitney's test for continuous data, and proportion measurements were analyzed using the Chi-square test. For comparing ROP degrees between groups, post hoc analysis involved pairwise comparisons using multiple z-tests of two-proportion with a Bonferroni correction, and statistical significance was accepted at $p<0.006$. A logistic regression analysis was performed to verify the influence of variables previously known by their relationship with BPD development (such as PMA at birth, birth weight, gender and diagnosis of $\mathrm{PH}$ ), as well as time under MV found by the ROC curve in the development of moderate to severe BPD. Variables that showed standard deviation greater than 2.5 in residual analysis were excluded. In order to validate the cut-off point found by ROC curve, Chi-square test was performed on a different sample $(n=16)$ from the previously analyzed, applying the same eligibility, inclusion and exclusion criteria of the primary analysis with patients born in 2018 and 2019, using the time under MV above and below the cutoff point in each group. The statistical significance adopted was $p<0.05$.

\section{Results}

Of the 112 preterm infants born at $\leq 28$ weeks PMA admitted to the unit, 41 died before completing 28 days of life and 2 were not diagnosed with BPD. Among those included in the study, 16 were excluded: 5 did not need MV during hospitalization and 11 died before 36 weeks PMA, making BPD classification not possible (Fig. 1). Data such as PMA at birth, weight, gender, Apgar score, MV time and length of stay of patients included in the study are described in Table 1.

The cutoff point for time under MV identified as the best predictor for moderate to severe BPD development identified on the ROC curve was 36 days ( $75 \%$ sensitivity [95\% CI 55 to 89] and $76 \%$ specificity [95\% CI 55 to 91]). Area under the curve was 0.83 (Fig. 2).

The comparison between the groups of preterm infants with mild BPD and those with moderate to severe BPD can be found in Table 2. There was no difference between groups regarding maternal and gestational characteristics. Both groups were similar in weight, PMA at birth, Apgar score and respiratory support needed in the delivery room, but the moderate to severe BPD group had a higher proportion of males.

Regarding the variables during hospitalization, preterm infants in moderate to severe BPD group had a longer time under MV, lower rates of CPAP use before intubation, higher rates of pulmonary hypertension, and greater severity of ROP. At discharge, individuals in the moderate to severe BPD group had been hospitalized longer and had a higher weight and were older than those in the mild BPD group.

Logistic regression which verified the influence of different clinical variables in the development of moderate to severe BPD was statistically significant $(p<0.0005)$. The model explained $72 \%$ of the variance in moderate to severe BPD and correctly classified $86 \%$ of the cases. After checking for residuals, three subjects were excluded from the analysis. Only two out of the five predictor variables were statistically relevant: $\mathrm{PH}$ and time under MV greater than 36 days (Table 3). Preterm infants who remained under MV for more than 36 days were 49 times more likely to develop moderate to severe BPD than those who needed MV for less than 36 days. The diagnosis of $\mathrm{PH}$ was associated with 37 times increase in chances of developing moderate and severe BPD. 


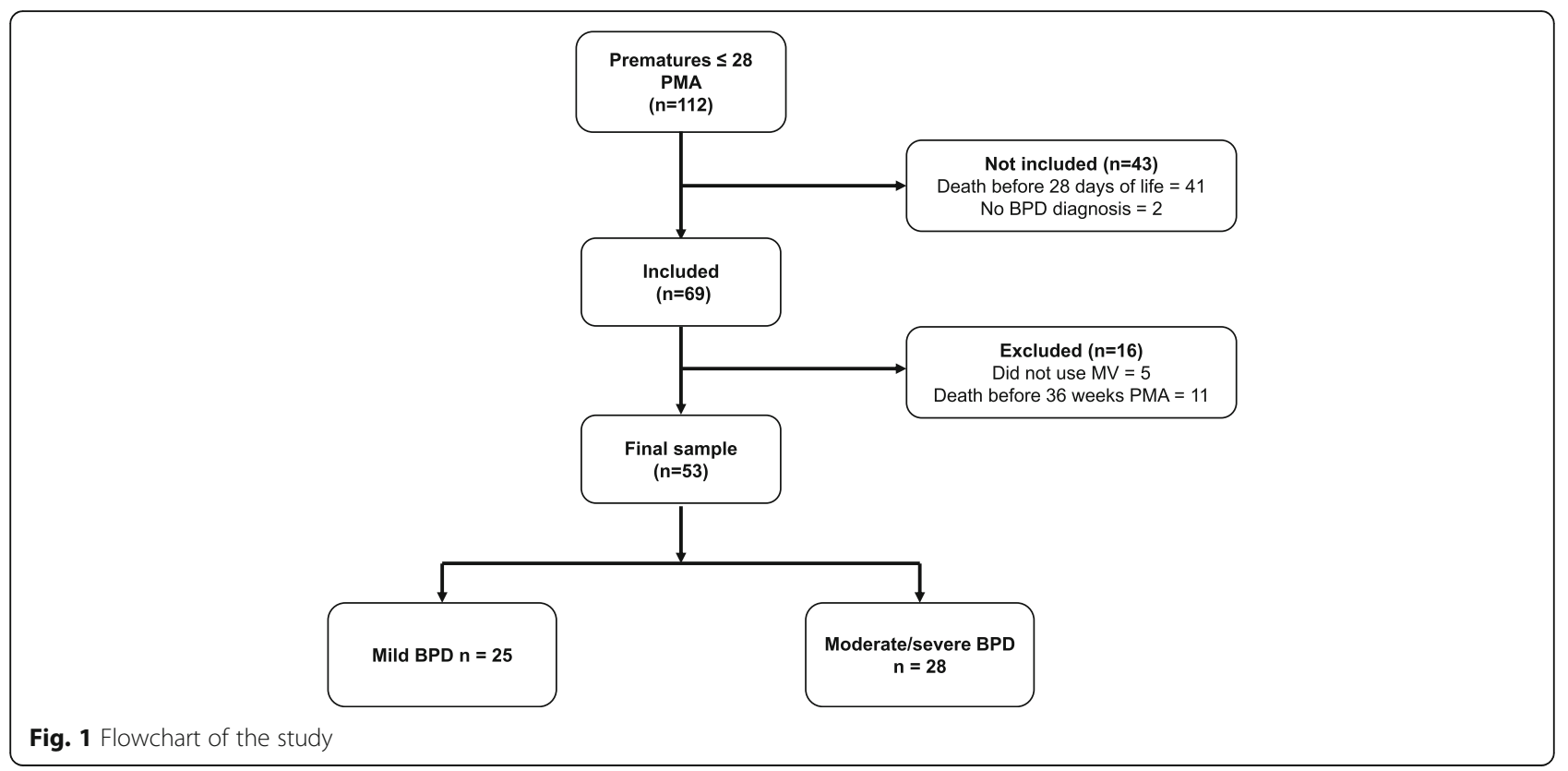

In the validation sample, of the 16 preterm included in the analysis, 10 had mild BPD and 6 moderate to severe BPD. The prevalence of preterm infants who were under MV for more than 36 days in the moderate to severe BPD group was $100 \%(n=6)$, and $0 \%$ in the mild BPD group $(p=0.0001)$.

\section{Discussion}

The present study showed that there is greater chance of developing moderate to severe BPD in extremely premature newborns after 36 days under mechanical ventilation [16]. It is important though to make it clear that BPD is a multifactorial disease, and its development is also associated to factors such as intrauterine infections, growth restriction and nicotine exposure [16]. Due to the pathophysiology of the disease, BPD remains the most frequent morbidity in extremely premature infant [17], and the diagnosis of moderate to severe BPD is associated with worse prognosis. A previous study comparing infants with different disease severity classifications showed that the group with moderate to severe BPD had a higher proportion of subjects with grade 3 and 4 intraventricular hemorrhage, periventricular leukomalacia, NEC, late sepsis, home oxygen use and death after discharge, in addition to the increased incidence of neurological impairment, worse mental and psychomotor development, blindness and hearing impairment [18]. In the present study, infants with moderate to severe BPD had worse ROP, higher prevalence of $\mathrm{PH}$, and longer length of hospital stay, corroborating the findings in the literature that preterm infants have worse outcomes.

Studies have shown a high incidence of moderate to severe BPD in extremely premature infants (PMA up to 27$29 \mathrm{w})$. Stoll et al. reported an incidence of $41 \%$ in their population [19]. Another study with extremely low birth weight infants $(401-1000 \mathrm{~g})$ found that $52 \%$ of the population had diagnosis of moderate to severe BPD [18]. In the present study, more than half of the preterm infants included (53\%) had moderate to severe BPD (Fig. 1).

Recent studies have enhanced the harms of exposure for extremely preterm infants to MV for long periods of time. Yossef et al. observed that a group of extremely premature infants that were submitted to more than 56 days of MV had a higher incidence of moderate to severe BPD [20]. Choi et al. found an almost three-fold increase in the risk of mortality of extremely low birth weight preterm infants who required MV for 15 to 28 days. Furthermore, that study also associated cumulative

Table 1 Characteristics of patients included in the study

\begin{tabular}{llll}
\hline Characteristics & $\begin{array}{l}\text { Mild BPD group } \\
(\boldsymbol{n}=25)\end{array}$ & $\begin{array}{l}\text { Moderate/severe BPD group } \\
(\boldsymbol{n}=28)\end{array}$ & $\boldsymbol{p}$ \\
\hline GA, weeks & $26,34 \pm 1,02$ & $25,92 \pm 1,37$ & 0,305 \\
Weight at birth, g & $830,5 \pm 150,26$ & $760,07 \pm 166,04$ & 0,119 \\
Gender, M (\%) & $5(20)$ & $14(50)$ & $0,047^{*}$ \\
Apgar 5' & $8[6-9]$ & $8[7-8]$ & 0,81 \\
\hline
\end{tabular}

GA gestational age, $g$ grams, $M$ male, Apgar $5^{\prime}$ Apgar score at fifth minute of life; ${ }^{*} p<0,05$. 


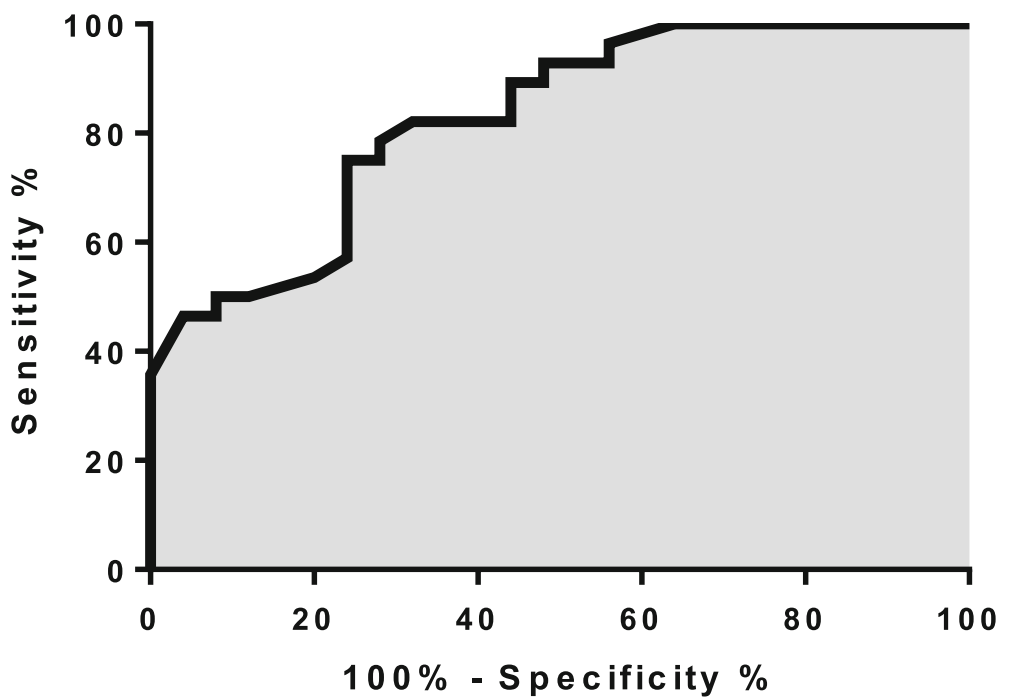

Fig. 2 Cut-off point of time under mechanical ventilation. ROC curve showing sensibility and specificity of time under MV with greater chance to develop moderate/severe BPD. Area under the curve 0,83

duration of MV with ROP requiring surgical correction, neurological impairment, BPD, PH, and length of stay [6]. The major cause of the development of ROP is the exposure of preterm infants to supplemental oxygen therapy at high concentrations, therefore its relationship with prolonged MV is already expected [11]. Although the number of days under MV found in our study is different from the aforementioned studies, our findings are mostly similar to the literature. It reinforces the importance to avoid invasive MV, as well as to limit the duration that the extremely preterm infants are exposed to this treatment.

The mild BPD group had more individuals who used CPAP before MV, and it is an expected result. In centers where CPAP is used during stabilization in the delivery room, the incidence of severe BPD is 3.3\% [21], and intubation for surfactant administration alone (without MV) has shown a significant reduction in the use of MV in extremely low birth weight preterm infants [22]. In addition, compared with intubation right after birth, CPAP reduces the incidence of BPD and death at 36 weeks of PMA [23].

Laughon et al. in their large study $(n=3629$ preterm infants) to develop an instrument for predicting BPD risk and death through clinical information, six variables were relevant to the model: PMA at birth, birth weight, ethnicity, gender, respiratory support and inspiratory oxygen fraction on specific days of hospitalization [10]. Factors such as PMA and weight were also related to longer time under $\mathrm{MV}^{20}$. In our search for predictors for development of moderate to severe BPD, in addition to time on MV greater than 36 days, the presence of $\mathrm{PH}$ appeared as significant in the regression model. It is associated with prolonged exposure to $\mathrm{MV}^{6}$, and the association between $\mathrm{PH}$ and BPD is related to both its pathogenesis (similarly associated to complications of prematurity) and secondary $\mathrm{PH}$ development in BPD [24]. There was no difference between groups regarding PDA, NEC, use of VAD, chorioamnionitis and maternal use of tobacco, unlike other studies about both prolonged time under MV and higher severity of BPD [6,20], which might have occurred due to the relatively small sample size. Additionally, a reasonable number of BPD patients did not survive until they completed 36 weeks PMA, which may also have contributed to this difference in outcomes.

Controversies regarding the definition and classification of BPD in literature may have limited our results. One premature newborn died before completing 36 weeks PMA and 41 died before 28 days of age. According to the most recent workshop on BPD, those infants could be classified as BPD type IIIa (early death from lung disease and respiratory failure) [25], and this could have influenced our findings. Our study involved preterm infants from a single institution and included a small sample compared to similar ones. Additionally, long-term outcomes such as neurodevelopment were not analyzed. However, almost all variables that may influence the development and severity of BPD were analyzed, except genetic factors. Despite the weaknesses, most of the findings of the present study, which is a pilot study, are in line with the findings from the literature.

Despite the limitations, it is noteworthy that this study is the first to investigate a cutoff point of time under MV to identify the chance of developing more severe forms of BPD. In addition, the result found was validated in an independent sample. Our study was able to define 36 days as a time of MV exposure that is associated with 
Table 2 Comparison between groups

\begin{tabular}{|c|c|c|c|}
\hline & Mild BPD & Moderate/severe BPD & $p$ \\
\hline \multicolumn{4}{|l|}{ Maternal and gestational characteristics } \\
\hline Tobacco use, yes (\%) & $2(8)$ & $4(14,3)$ & 0,672 \\
\hline Chorioamnionitis, yes (\%) & $3(12)$ & $5(18,5)$ & 0,705 \\
\hline Antenatal corticosteroids, yes (\%) & $23(92)$ & $20(74,1)$ & 0,143 \\
\hline \multicolumn{4}{|l|}{ Perinatal variables } \\
\hline Intubation in the delivery room, yes (\%) & $12(48)$ & $20(71,4)$ & 0,072 \\
\hline CPAP in the delivery room, yes (\%) & $13(52)$ & $8(28,6)$ & 0,99 \\
\hline \multicolumn{4}{|l|}{ Outcomes during hospitalization } \\
\hline Time under MV (days) & $19[7,25-38]$ & $43[33,25-52,75]$ & $<0,001^{*}$ \\
\hline CPAP before intubation, yes (\%) & $12(56)$ & $7(25)$ & $0,043^{*}$ \\
\hline RDS, yes (\%) & $13(76)$ & $24(85,7)$ & 0,488 \\
\hline Surfactant use, yes (\%) & $18(72)$ & $24(85,7)$ & 0,313 \\
\hline Pulmonary hypertension, yes (\%) & $3(12)$ & $12(42,9)$ & $0,016^{*}$ \\
\hline Pneumonia, yes (\%) & $23(92)$ & $28(100)$ & 0,218 \\
\hline \multicolumn{4}{|l|}{ ROP classification, n (\%) } \\
\hline 0 & $13(52)$ & $4(14,3)$ & 0,009 \\
\hline 1 & $6(16)$ & $11(39,3)$ & 0,23 \\
\hline 2 & $4(16,2)$ & $2(7,1)$ & 0,31 \\
\hline 3 & $2(8)$ & $11(39,3)$ & $0,003^{\mathrm{a}}$ \\
\hline Corrective surgery for ROP, yes (\%) & $1(5,6)$ & $4(15,4)$ & 0,634 \\
\hline PDA, sim (\%) & $22(88)$ & $24(85,7)$ & 1 \\
\hline Drug treatment for PDA, yes (\%) & $9(40,9)$ & $11(45,8)$ & 0,774 \\
\hline Surgery for PCA, yes (\%) & $2(6,5)$ & $1(4,2)$ & 0,6 \\
\hline NEC, yes (\%) & $3(12)$ & $3(10,7)$ & 1 \\
\hline Surgery for NEC, yes (\%) & $1(33,3)$ & $2(66,7)$ & 1 \\
\hline Sepsis, yes (\%) & $22(88)$ & $26(92,9)$ & 0,658 \\
\hline VAD, yes (\%) & $5(20)$ & $2(7,1)$ & 0,234 \\
\hline \multicolumn{4}{|l|}{ Variables at discharge } \\
\hline Lenght of stay (days) & $81,5[69,5-90,5]$ & $109[99-118,25]$ & $<0,001^{*}$ \\
\hline GA at discharge (weeks) & $38[36,25-38,75]$ & $41[39-42,75]$ & $<0,001^{*}$ \\
\hline Weight at discharge (g) & $2031[1868-2328]$ & $2620[2045-3030]$ & $<0,001^{*}$ \\
\hline $\mathrm{O}_{2}$ discharge/transference, yes (\%) & $0(0)$ & $4(14,3)$ & 0,115 \\
\hline
\end{tabular}

GA gestational age, $g$ grams, Apgar $5^{\prime}$ Apgar score at fifth minute of life, $M$ male, $M V$ mechanical ventilation, CPAP continuous positive airway pressure, $R D S$ respiratory distress syndrome, $R O P$ retinopathy of prematurity, $P D A$ patente ductus arteriosus, NEC necrotizing enterocolits, VAD vasoactive drugs, $O_{2}$ oxygen; ${ }^{*}$ $p<0,05 ;{ }^{a} p<0,006$

the development of moderate to severe BPD and, as a consequence, may have worse outcomes, such as greater severity of ROP and length of hospital stay. Therefore, it is suggested that MV should be avoided whenever possible, using strategies such as prophylactic CPAP and less invasive surfactant admnistration [26, 27]. Moreover, MV should be interrupted as soon as possible, preferably before 36 days to reduce the risk of developing moderate to severe BPD, as well as to avoid impairments associated with the severity of the disease. Furthermore, a multicenter study should be performed to verify whether similar results are observed with a larger sample of patients. Indeed, this characterizes the next step for a future study in which we will
Table 3 Logistic Regression predicting likelihood of developing moderate/severe BPD based on MV > 36 days, GA, PH, weight and gender

\begin{tabular}{llllll}
\hline & & & & \multicolumn{2}{c}{$95 \%$ C.I. for EXP } \\
\cline { 6 - 7 } Variable & B & $\boldsymbol{p}$ & RR & Lower & Upper \\
\hline Constant & -7528 & 0,506 & 0,001 & & \\
MV $>36$ days & 3894 & 0,001 & 49,101 & 4916 & 490,415 \\
GA (days) & 0,048 & 0,469 & 1050 & 0,921 & 1196 \\
PH & 3632 & 0,011 & 37,770 & 2270 & 628,541 \\
Weight at birth & $-0,005$ & 0,125 & 0,995 & 0,988 & 1001 \\
Gender & 2014 & 0,143 & 7497 & 0,506 & 110,976 \\
\hline
\end{tabular}

MV Mechanical ventilation, GA gestational age, $P H$ Pulmonary hypertension, $B$ nonstandard coefficients regression, $R R$ relative risk, $C . I$ confidence interval. Note: Gender is for males compared to females 
include other hospitals from the Brazilian neonatal research network.

\section{Conclusion}

In conclusion, we can state that the duration of MV exposure associated with the development of moderate to severe BPD was 36 days, and worse outcomes such as worse ROP and longer hospital staying are associated with disease severity.

\section{Abbreviation}

BPD: Bronchopulmonary dysplasia; MV: Mechanical Ventilation; GA: Gestational age; ROP: Retinopathy of prematurity; CPAP: Continuous positive airway pressure; VAD: Vasoactive drugs; PDA: Patent ductus arteriosus; PH: Pulmonary Hypertension; NEC: Necrotizing enterocolitis; AUC: Area under the curve

\section{Acknowledgements}

We thank the team of professionals at the Neonatal ICU at Londrina State University Hospital for their efforts in collecting the data and feeding the database.

\section{Authors' contributions}

VE: literature search, data collection, study design, data analysis, manuscript preparation, review of manuscript. DS: data collection, review of manuscript. JK: data collection, study design, review of manuscript. LF: data collection, review of manuscript. JMF: study design, review of manuscript. CAMC: study design, data analysis, review of manuscript. VSP: literature search, study design, data analysis, manuscript preparation, review of manuscript. All authors have read and approved the manuscript.

\section{Funding}

This study was financed in part by the Coordenação de Aperfeiçoamento de Pessoal de Nível Superior - Brasil (CAPES) - Finance Code 001

\section{Availability of data and materials}

The datasets used and/or analysed during the current study are available from the corresponding author on reasonable request.

\section{Ethics approval and consent to participate}

Research Ethics Commitee Involving Human Beings - Londrina State University - Brazil.

Commitee's reference number: 3.362 .155 .

Since there was no direct approach with individuals but only with medical records, the Research Ethics Commitee Involving Human Beings from Londrina State University waived the use of the informed consent. However, due to data manipulation, the commitee requested a term of confidentiality signed by the main reseacher, in order to guarantee data privacy.

\section{Consent for publication}

Not Applicable.

\section{Competing interests}

The authors declare that they have no competing interests.

\begin{abstract}
Author details
${ }^{1}$ Neonatal Intensive Unit, University Hospital, Londrina State University (UEL), Londrina, PR, Brazil. ${ }^{2}$ Postgraduation Program in Rehabilitation Sciences UEL-UNOPAR, Londrina State University (UEL), Londrina, PR, Brazil. ${ }^{3}$ Pediatric Surgery and Pediatric Department, Center of Health Sciences, Londrina State University (UEL), Londrina, PR, Brazil. ${ }^{4}$ Physiotherapy Department, Center of Health Sciences, Londrina State University, 60 Robert Koch Av, Londrina, Parana 86038-350, Brazil. ${ }^{5}$ Pitagoras Unopar University, Londrina, PR, Brazil.
\end{abstract}

Received: 10 February 2020 Accepted: 6 May 2020

Published online: 21 May 2020

\section{References}

1. Jobe AH, Steinhorn R. Can we define Bronchopulmonary dysplasia? J Pediatr. 2017;188:19-23.
2. Jobe $A H$, Bancalari E. Bronchopulmonary dysplasia. Am J Respir Crit Care Med. 2001;163(7):1723-9.

3. Isayama T, Lee SK, Yang J, et al. Revisiting the definition of Bronchopulmonary dysplasia: effect of changing panoply of respiratory support for preterm neonates. JAMA Pediatr. 2017;171(3):271-9.

4. Organization WH. ICD-11 international classification of diseases 11th revision - the global standard for diagnostic health information. 2018.

5. Stoll BJ, Hansen NI, Bell EF, et al. Trends in care practices, morbidity, and mortality of extremely preterm neonates, 1993-2012. Jama. 2015;314(10):1039-51.

6. Choi YB, Lee J, Park J, Jun YH. Impact of prolonged mechanical ventilation in very low birth weight infants: results from a National Cohort Study. J Pediatr. 2018;194:34-9 e33.

7. Miller JD, Carlo WA. Pulmonary complications of mechanical ventilation in neonates. Clin Perinatol. 2008;35(1):273-81 x-xi.

8. Walsh MC, Morris BH, Wrage LA, et al. Extremely low birthweight neonates with protracted ventilation: mortality and 18-month neurodevelopmental outcomes. J Pediatr. 2005;146(6):798-804.

9. Carvalho CG, Silveira RC, Procianoy RS. Ventilator-induced lung injury in preterm infants. Revista Brasileira de terapia intensiva. 2013;25(4):319-26.

10. Laughon $\mathrm{MM}$, Langer $\mathrm{JC}$, Bose $\mathrm{CL}$, et al. Prediction of bronchopulmonary dysplasia by postnatal age in extremely premature infants. Am J Respir Crit Care Med. 2011;183(12):1715-22.

11. Quimson SK. Retinopathy of prematurity: pathogenesis and current treatment options. Neonatal network: NN. 2015:34(5):284-7.

12. Bell MJ, Ternberg JL, Feigin RD, et al. Neonatal necrotizing enterocolitis. Therapeutic decisions based upon clinical staging. Ann Surg. 1978;187(1):1-7.

13. Wynn JL, Wong HR, Shanley TP, Bizzarro M, Saiman L, Polin RA. Time for a neonatalspecific consensus definition for sepsis. Pediatric Critical Care Med. 2014;15(6):523-8.

14. Youden WJ. Index for rating diagnostic tests. Cancer. 1950;3(1):32-5.

15. Metz CE. Basic principles of ROC analysis. Semin Nucl Med. 1978;8(4):283-98.

16. Jobe AH. Mechanisms of lung injury and Bronchopulmonary dysplasia. Am J Perinatol. 2016;33(11):1076-8.

17. Aschner JL, Bancalari EH, McEvoy CT. Can we prevent Bronchopulmonary dysplasia? J Pediatr. 2017;189:26-30.

18. Natarajan G, Pappas A, Shankaran S, et al. Outcomes of extremely low birth weight infants with bronchopulmonary dysplasia: impact of the physiologic definition. Early Hum Dev. 2012;88(7):509-15.

19. Stoll BJ, Hansen NI, Bell EF, et al. Neonatal outcomes of extremely preterm infants from the NICHD neonatal research network. Pediatrics. 2010;126(3):443-56.

20. Yossef L, Shepherd EG, Lynch S, Reber KM, Nelin LD. Factors associated with long-term mechanical ventilation in extremely preterm infants. J NeonatalPerinatal Med. 2018;11(1):29-35.

21. Ammari A, Suri M, Milisavljevic $V$, et al. Variables associated with the early failure of nasal CPAP in very low birth weight infants. J Pediatr. 2005;147(3):341-7.

22. Sekar KC, Corff KE. To tube or not to tube babies with respiratory distress syndrome. J Perinatol. 2009:29(Suppl 2):S68-72.

23. Foglia EE, Jensen EA, Kirpalani H. Delivery room interventions to prevent bronchopulmonary dysplasia in extremely preterm infants. J Perinatol. 2017; 37(11):1171-9.

24. Wright MFA, Wallis C. Investigation and management of the long-term ventilated premature infant. Early Hum Dev. 2018;126:10-7.

25. Higgins $\mathrm{RD}$, Jobe $\mathrm{AH}$, Koso-Thomas $\mathrm{M}$, et al. Bronchopulmonary dysplasia: executive summary of a workshop. J Pediatr. 2018;197:300-8.

26. Subramaniam P, Ho JJ, Davis PG. Prophylactic nasal continuous positive airway pressure for preventing morbidity and mortality in very preterm infants. The Cochrane database of systematic reviews. 2016;6:CD001243.

27. Langhammer K, Roth B, Kribs A, Gopel W, Kuntz L, Miedaner F. Treatment and outcome data of very low birth weight infants treated with less invasive surfactant administration in comparison to intubation and mechanical ventilation in the clinical setting of a cross-sectional observational multicenter study. Eur J Pediatr. 2018;177(8):1207-17.

\section{Publisher's Note}

Springer Nature remains neutral with regard to jurisdictional claims in published maps and institutional affiliations. 